\title{
Effects of Early Musical Experience on Auditory Sequence Memory
}

\author{
ADAM T. TIERNEY \\ Department of Cognitive Science, UC San Diego \\ TONYA R. BERGESON \\ Department of Otolaryngology, Indiana University School of Medicine \\ DAVID B. PISONI \\ Department of Psychological and Brain Science, Indiana University
}

\begin{abstract}
The present study investigated a possible link between musical training and immediate memory span by testing experienced musicians and three groups of musically inexperienced subjects (gymnasts, Psychology 101 students, and video game players) on sequence memory and word familiarity tasks. By including skilled gymnasts who began studying their craft by age six, video game players, and Psychology 101 students as comparison groups, we attempted to control for some of the ways skilled musicians may differ from participants drawn from the general population in terms of gross motor skills and intensive experience in a highly skilled domain from an early age. We found that musicians displayed longer immediate memory spans than the comparison groups on auditory presentation conditions of the sequence reproductive span task. No differences were observed between the four groups on the visual conditions of the sequence memory task. These results provide additional converging support to recent findings showing that early musical experience and activity-dependent learning may selectively affect verbal rehearsal processes and the allocation of attention in sequence memory tasks.
\end{abstract}

Submitted 2008 August 2; accepted 2008 September 24.

KEYWORDS: Skilled musicians, memory span, sequence learning

MUSIC practice involves the development of a vast array of perceptual, cognitive, and motor skills, including the assembly and memorization of long sequences of motor commands, extremely precise motor timing, fine pitch discrimination, and memorization of hours of auditory information. This experience differs in several significant ways from the training that a typical elementary school student receives. Nevertheless, the question of whether the development of these skills may have consequences outside the musical sphere has not been widely addressed. Recently, however, several researchers have argued that musical training and experience facilitate the development of verbal skills, especially verbal memory (Chan, Ho, \& Cheung, 1998; Ho, Cheung, \& Chan, 2003; Jakobson, Cuddy, \& Kilgour, 2003). In the present study, we attempted to determine whether musicians would show larger verbal memory spans even when compared to gymnasts, a group of people who also, at a young age, took part in a highly structured activity involving repeated practice of certain motor movements over a long period of time. In addition, we tested students who played video games daily, a group that practices daily a skill involving the control of fine finger movements and focused attention.

Research has shown that early experience with music can have selective effects on brain development that are strong enough to be detectable in the physical brain structure. In 1995, for example, Schlaug, Jancke, Huang, and Steinmetz, using MRI, found that a group of musicians with perfect pitch had a greater disparity between the left and right planum temporale (PT), an area of the brain that is involved in music perception, as compared to nonmusicians and musicians with relative pitch. The authors speculated that this increase in left-right disparity might indicate increased lateralization of music processing in musicians with absolute pitch, as compared to non-musicians or musicians with relative pitch.

In a follow-up study, Schlaug, Jancke, Huang, Staiger, and Steinmetz (1995b) reported that the midsagittal area of the anterior half of the corpus callosum was larger in a group of musicians who had begun playing piano and stringed instruments by age seven than in a group of non-musicians, a result most likely due to the need for increased coordination between the two hands. Using fMRI, Elbert, Pantev, Wienbruch, Rockstroh, and Taub (1995) found that string musicians had increased cortical representation of the fingers of their left hands, as compared to 
non-musicians. This finding was correlated with the age at which each musician began to play music. Finally, using fMRI, Schneider et al. (2002) found that musicians had a significantly greater volume of gray matter in Heschl's gyrus, especially in primary auditory cortex when compared to non-musicians. Given the findings that early experience with music can produce measurable neurobiological changes, it is reasonable to ask what differences in cognitive skills, especially in the auditory domain, musicians might show when compared to non-musicians.

One of the first researchers to investigate the relation between musical and non-musical cognitive skills was McMahon (1982). He found a strong positive correlation between performance on the Enticknap Picture Vocabulary Test, which requires children to name line drawings of objects on 48 test cards, and performance on a tonal discrimination test designed by the experimenter. Additional correlations between verbal and musical skills were uncovered by Atterbury (1985), who reported that learning-disabled readers performed significantly worse than typical readers on tonal discrimination and rhythmic discrimination tasks. Barwick, Valentine, West, and Wilding (1989) also found significant positive correlations between tonal memory skills and reading age, even after adjusting for chronological age and IQ. Finally, Lamb and Gregory (1993) reported significant positive correlations between a pitch discrimination test and tests that required children to read nonsense words aloud or demonstrate an understanding of rhyme and alliteration.

These correlational studies suggest that basic musical abilities, such as tone, rhythm, and chord discrimination, are associated with reading ability. However, the children with better musical skills may also possess better language skills overall simply because children with higher socioeconomic status and a more intellectually stimulating home environment are exposed more often to certain aspects of language and music (their parents may read to them more often, for example). In an effort to move beyond mere correlation and determine whether or not early musical experience actually affects subjects' non-musical cognitive skills, several researchers have turned to quasi-experimental studies in which subjects are divided into (at least) two groups: those with musical experience and those without.

In the first study of this kind, Huntsinger and Jose (1991) found that musically experienced children performed better than musically inexperienced children on tonal memory and digit span tasks. Similarly, Chan, Ho, and Cheung (1998) reported that musicians recalled more words from a visually presented 16-word list than nonmusicians. Kilgour, Jakobson, and Cuddy (2000) asked undergraduate musicians and non-musicians to memorize lyrics that were either spoken or sung, one line at a time. The musicians showed better recall of both the sung and spoken lyrics. Munzer, Berti, and Pechmann (2002) also found that musicians performed better than non-musicians on a task which required them to decide if the first and last stimuli in a set of speech sounds were the same or different.

Jakobson, Cuddy, and Kilgour (2003) attempted to delve further into the reasons why music training leads to enhanced verbal recall. They compared musicians and non-musicians on tests of verbal recall and temporal order processing, in which the subjects were asked to determine the order of presentation of two tones or two syllables of equal duration. The correlation between years of musical training and verbal recall scores was strong and significant, as was the correlation between musical training and the composite score for the temporal order tests. Using a multiple-regression analysis, the authors found that the relation between verbal recall scores and years of music training was reduced when temporal-order processing was included in the regression equation. Thus, it appears that the advantage that musicians show in verbal recall tasks is selective in nature and may be due in part to their enhanced temporal order processing abilities and skills in encoding temporal sequences. Finally, Ho, Cheung, and Chan (2003) found that a year of piano instruction led to improved verbal recall scores, as compared to a group that received no instruction. However, the study was not truly experimental because participation in music training was decided on a voluntary basis; that is, the authors examined students who continued and those who dropped out of musical instruction.

One cannot infer causation from any of these studies because it is possible that children with greater cognitive skills in the verbal/auditory domain are simply more likely to be drawn to music and that this predilection could account for the musicians' better performance on verbal memory tasks. In order to establish whether or not the better performance on reading and verbal memory tasks results from musical training, a truly experimental study with randomly selected experimental and control groups is needed. Only one such study has thus far been attempted. Schellenberg (2004) provided children with a year of piano instruction, a year of drama lessons, or no instruction at all, and found that the children who received piano instruction showed significantly greater increases in IQ than the control groups; this difference was found on all but two of the twelve subtests of the WISC-III IQ test (Wechsler, 1991).

Only truly experimental studies, such as Schellenberg (2004), avoid confounds due to differences between musicians and non-musicians unrelated to musical experience. However, it is not practical for truly experimental studies to examine the effect of the thousands of hours of practice that musicians undergo before achieving mastery 
of their instruments. One way to tap into the advantages of the quasi-experimental approach while accounting for some of the possible differences between musicians and non-musicians is to test the verbal skills of a comparison group that has practiced a skilled motor task intensively from an early age. In the present study, we measured performance of skilled musicians from the Indiana University School of Music on a test of verbal and visuospatial memory span. The verbal and visuospatial memory tests required subjects to respond in identical ways, and thus any difference in performance between these two tests could only be attributed to differences in input processing and prior experience. We also tested subjects from three comparison groups, all of whom were unable to read music: (a) gymnasts from the IU Gymnastics Club, (b) students who played video games for at least 1.5 hours per day, and (c) Psychology 101 students at IU. We hypothesized that musicians' greater ability to focus and maintain attention on auditory stimuli would facilitate their performance on the auditory-only memory task, but that performance on the visual-only task would not differ between the groups. The students who intensively played video games daily were included as a comparison group because these students - like the music majors - engaged in daily practice of an activity requiring fine finger movements and focused attention.

\section{METHODS}

\section{Subjects}

Forty-five students participated in the study. A summary of the demographic characteristics of the participants is given in Table 1. By sending e-mails to the distribution list of the IU Gymnastics Club, we recruited ten gymnasts who could not read music and began gymnastics, on average, when they were 4.5 years of age. (None of the gymnasts started practicing later than age six.) They practiced an average of 4.5 hours per week. We also recruited 12 experienced students from the IU School of Music who were piano performance majors by sending e-mails to the distribution list for all of the undergraduate and graduate piano performance majors. The mean age at which the pianists began playing the piano was 7 years. The average number of hours they spent practicing every week was 24.18. Twelve students were undergraduates randomly drawn from the pool of students taking introductory psychology courses at IU who could not read music. The psychology students were contacted via a list of students who indicated on a questionnaire at the end of class that they wished to participate in paid psychology experiments. Finally, we recruited 11 students who played video games for at least 10.5 hours per week, and were unable to read music. The ability to read music was used as the criterion distinguishing "musician" from "non-musician" in order to investigate possible links between musical training and memory span. While some of the non-musician subjects may have attained a certain level of musical skill without gaining the ability to read music, it is rather unlikely that they could have gone through any significant musical training regimen without acquiring that ability. The difference in the ages among the four groups at the time of testing was not statistically significant $(p>.05)$. All subjects were paid $\$ 15$ for their time.

\begin{tabular}{lccc} 
& Age & Num. Males & Num. Females \\
\hline Gymnasts & $19.9($ SD 1.4) & 0 & 10 \\
Musicians & 21.1 (SD 2.3) & 7 & 5 \\
Non-musicians & 19.4 (SD 1.2) & 2 & 10 \\
Video gamers & 22.3 (SD 3.1) & 11 & 0
\end{tabular}

Table 1. Demographic data for gymnasts, musicians, Psychology non-musicians and video game players.

\section{Stimulus Materials}

All subjects completed a sequence reproduction task and a word familiarity task. The test words for the wordfamiliarity (FAM) vocabulary test were taken from Stallings, Kirk, Chin, and Gao (2000), a shortened version of the FAM test originally developed by Lewellen, Goldinger, Pisoni, and Greene (1993). The 150-word stimulus list consisted of 50 high-familiarity words, 50 medium-familiarity words, and 50 low-familiarity words. The familiarity 
scores were based on normed familiarity ratings of 20,000 words collected originally by Nusbaum, Pisoni, and Davis (1984).

\section{Experimental Design and Procedures}

\section{SEQUENCE REPRODUCTION TASK}

This task used a modified version of the Simon Memory Game, a round box with four colored response panels that was interfaced to a computer (Karpicke \& Pisoni, 2004; Pisoni \& Cleary, 2004). The panels of the box were colored red, green, yellow, and blue. In the first condition, visual-only (VO), the colored panels were illuminated, one at a time, in a random sequence. The subjects were then asked to reproduce the sequence of colored lights by pressing the appropriate colored panels on the box. In the second condition, auditory-only (AO), the subjects heard a random sequence of the four color names over their headphones. They were asked to reproduce the sequence of color names by pressing the appropriate colored panels on the box. In the final condition, audiovisual (AV), the response panels on the box were illuminated and the subjects simultaneously heard names of colors that corresponded to the colors of the lights. They recorded their responses by pressing on the colored panels. The lights remained on for the duration of each auditory stimulus. In each of the three presentation conditions, the sequence length started out at one item, then increased by one whenever the subjects correctly reproduced a particular sequence length twice in a row. If the subjects reproduced a sequence incorrectly, the sequence length decreased by one item. Each condition (VO, AO, and AV) consisted of twenty trials.

\section{WORD FAMILIARITY RATINGS}

This is a test of subjective familiarity using high, medium, and low familiarity words. Fifty words from each category were presented randomly on a computer screen, one at a time, for a total of 150 words. The subjects were asked to judge the familiarity of the word by pressing the appropriate number key on the keyboard, from " 1 " for least familiar to "7" for most familiar.

\section{RESULTS}

\section{Sequence Memory Spans}

Scores on the sequence span task were calculated by taking a weighted sum of the percentage of correct responses at each list length (see Pisoni \& Cleary, 2004). Figure 1 shows mean weighted span scores for the four groups of subjects in each of the three different presentation conditions (VO, AO, and AV); musicians outperformed the comparison groups in the $\mathrm{AO}$ condition, but no difference in performance was found in either the VO or $\mathrm{AV}$ conditions.

\section{Sim on Span}

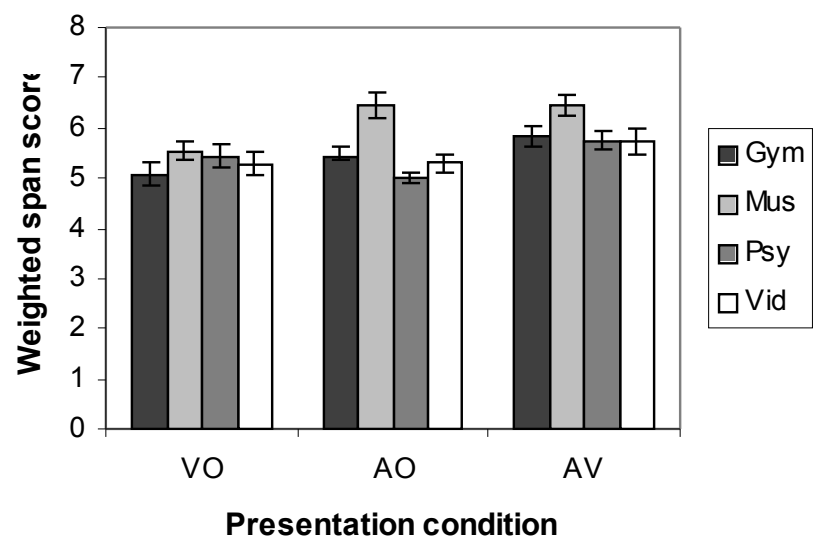


Fig. 1. Mean weighted span scores for the four groups of subjects in the three different presentation conditions ( $\mathrm{VO}, \mathrm{AO}$, and $\mathrm{AV})$ of the sequence memory span task. Error bars represent standard error.

The sequence span scores were entered into a repeated measures ANOVA, with group as a betweensubjects variable and presentation condition $(\mathrm{VO}, \mathrm{AO}$, and $\mathrm{AV})$ as a within-subjects variable. Main effects of presentation condition $\left[F(2,82)=16.88, M S_{e}=4.307, p<.001\right]$ and subject group $\left[F(3,41)=4.565, M S_{e}=4.548, p\right.$ $=.008]$ were statistically significant. A significant interaction between presentation condition and group $[\mathrm{F}(6,82)=$ 3.803, $M S_{e}=.970, p=.002$ ] was also found. Post hoc Bonferroni tests revealed that, overall, subjects did not perform better on the AO condition than on the VO condition $(p=.11)$. However, subjects did show significantly better performance on the AV condition as compared to the VO condition $(p<.001)$ and the AO condition $(p<$. 001). Further analysis revealed that the difference between the AO and AV conditions was only significant for the psychology subjects $(p=.002)$.

Thus, all four groups showed a "redundancy gain" (i.e., AV > VO). However, the difference between the $\mathrm{VO}$ and AO conditions was significant only for the musicians $(p=.011)$. Thus, among the four groups of subjects, only the musicians showed a "modality effect"; that is, they were able to reproduce auditory sequences (AO) better than visual sequences (VO). None of the pairwise differences in $\mathrm{VO}$ were significantly different. For the $\mathrm{AO}$ condition, the musicians (mean score $6.48, S D=.92$ ) outperformed the gymnasts (mean score $5.43, S D=.63, p=$ 0.006 ), the psychology students (mean score $5.02, S D=.33, p<0.001$ ), and the video game players (mean score $5.29, \mathrm{SD}=.65, p=.001$ ). No significant difference in performance was found between groups for the AV condition, although there was a trend for the musicians to display longer spans in this condition too.

\section{Word Familiarity Ratings}

Figure 2 shows mean familiarity ratings for the four groups of subjects in each of the three different word familiarity conditions (high-familiarity, medium-familiarity, and low-familiarity). The FAM ratings were entered into a repeated measures ANOVA, with group as a between-subjects variable and word familiarity (low, medium, and high) as a within-subjects variable. A main effect of familiarity $\left[F(2,60)=767.037, M S_{e}=168.290, p<.001\right]$ was found. Post-hoc analysis revealed no significant differences between the four groups. Musicians were not more likely to rate words as more familiar than any of the three comparison groups.

FAM Vocabulary Test

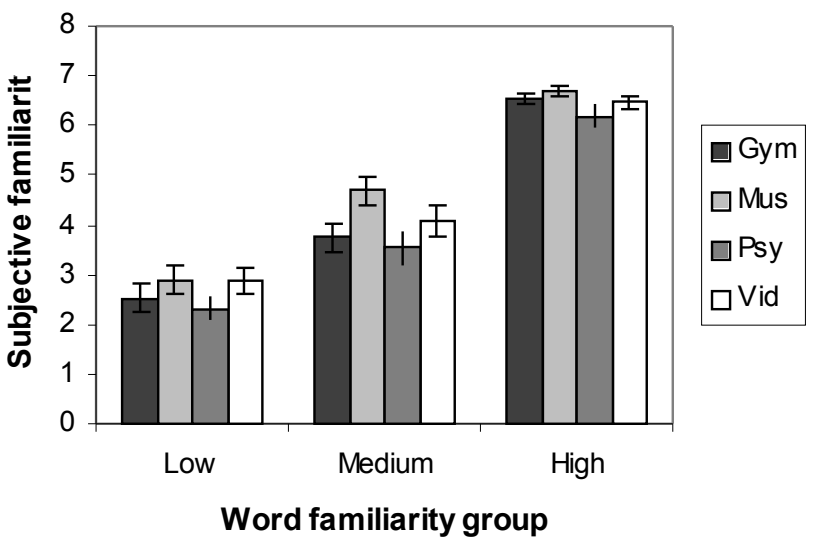

Fig. 2. Mean subjective familiarity ratings for the four groups in the FAM rating task. Error bars represent standard error.

\section{DISCUSSION}

Skilled musicians performed significantly better than the gymnasts, the introductory psychology students, and the video game players on the auditory-only (AO) condition of the sequence memory task. No significant differences 
were found between the groups on the visual-only (VO) condition or the audio-visual (AV) condition of this task. These findings suggest that skilled musicians display a greater selective capacity for reproducing randomized auditory sequences than non-musicians. The present results on auditory sequence memory span provide a counterargument to those who would claim that any cognitive differences between skilled musicians and nonmusicians is simply due to general intelligence, or to global differences in academic achievement. Effects of general intelligence and education are not likely to be so selective in nature and limited to a single processing domain or sensory modality (Gardner 1993). Moreover, the selective facilitation for the musicians in the AO condition cannot be due to differences in motor skills or task requirements, because the output for each of the three presentation conditions involved exactly the same response demands: pressing a sequence of four colored buttons on the response box. The only difference between the three conditions was the presentation modality of the stimulus sequences. Finally, the finding that the musicians did not score better than the other three groups on the word familiarity task suggests that, at least according to one measure indexing vocabulary knowledge, the four groups had received a similar degree of verbal academic training and exposure.

The sequence memory data obtained from the skilled musicians for the three presentation conditions also showed patterns that were different from those found in previous sequence memory and learning experiments that used groups of unselected introductory psychology students from the general university population. For example, Pisoni and Cleary (2004) reported results obtained in several studies that tested normal-hearing adults and normalhearing 8- and 9-year-old children on the AO, VO, and AV conditions of the sequence memory task. While children performed worse overall than adults, both groups showed two significant effects. First, both adults and children displayed "modality effects": that is, performance was better on AO than on VO. Secondly, both adults and children exhibited "redundancy gains": performance was better in the AV condition than in the VO condition. These two findings suggest that sequence memory spans are not only sensitive to input modality but also display cross-modal interactions between correlated auditory and visual dimensions. The same pattern of results was replicated recently by Karpicke and Pisoni (2004) in a study on implicit learning of temporal patterns using the same methodology.

In contrast to Pisoni and Cleary's earlier findings with adults and normal-hearing children, the skilled musicians in the present study showed a strong redundancy gain when the AV condition was compared to the VO condition, but no redundancy gain when the AV condition was compared to the $\mathrm{AO}$ condition. In fact, musicians actually performed slightly worse on the AV condition than on the AO condition, although this difference was not significant. Of the four groups tested in this experiment, only the skilled musicians exhibited a significant modality effect on the Simon sequence memory task, with performance on AO greater than performance on VO, although the gymnasts showed a trend in the same direction. When performance was averaged across all four groups, we did find an overall redundancy gain (that is, performance on $\mathrm{AV}$ was better than on $\mathrm{VO}$ and $\mathrm{AO}$ ), replicating the earlier findings of Pisoni and Cleary (2004) and Karpicke and Pisoni (2004). However, we failed to observe a significant overall modality effect across all four groups. Pisoni and Cleary (2004) found strong modality effects, but they used 48 subjects in their original study; the present study used a slightly smaller number of subjects. (See also Karpicke and Pisoni 2004).

Although we replicated the redundancy gains reported earlier by Pisoni and Cleary (2004) and Karpicke and Pisoni (2004) and found a trend towards a modality effect, skilled musicians displayed no benefit from the redundant visual information presented in the AV condition as compared with the $\mathrm{AO}$ condition. The other three groups of subjects all showed evidence of facilitation of performance in the AV condition when compared to the AO condition (i.e. redundancy gain), but this trend reached significance only for the psychology subjects, replicating the earlier findings. Apparently, auditory sequence information was so salient to the musicians that adding redundant visual information to the pattern provided little additional benefit.

The findings of the present study are analogous to those of Keehner and Gathercole (2006), who found that subjects who learned sign language as adults were more proficient at a visuospatial working memory task. This adaptation, however, was domain specific: their performance did not differ from that of control subjects on the regular Corsi block tapping task, but was enhanced when they were asked to rotate the blocks they were seeing by 180 degrees. (During normal conversation signers often need to rotate the movements that they are seeing 180 degrees in order to see the movements from the signer's point of view.) Similarly, in the present study, we found that experience with music was linked to an enhancement in auditory-verbal working memory, but had no effect on visuo-spatial working memory.

The present study used a quasi-experimental design, and thus could at best only uncover non-causal relations between musical experience and non-musical cognitive skills. Unfortunately, it is not possible to conduct a true experimental study of the effects of the amount of musical training which even good amateur musicians receive - playing music several hours daily for eight or nine years. To study the effects of musical experience, one must therefore either randomly assign a group of children to music lessons for a year or two as Schellenberg (2004) has 
done and risk underestimating the effect of more prolonged musical training, or compare trained musicians to several different comparison groups who are not musically trained but, ideally, are closely matched with the musicians in various ways. Each approach has its weaknesses, and neither approach on its own is sufficient to provide a definitive answer to the question of whether prolonged musical experience affects non-musical cognition.

Several studies have found, in contrast with our results, that musical experience was associated with increased visuospatial abilities, in the absence of any effects on verbal abilities. Hassler et al (1985), for example, compared the performance of children between the ages of nine and fourteen with and without musical experience on the Hidden Pattern Test, which tests visualization skills, and tests five and six of Horn's Leistungsprufsystem of general intelligence, which was used to assess verbal fluency. The authors found a significant relationship between musical talent and visualization, but not between musical talent and verbal ability. However, testing verbal fluency only examines one of a large number of diverse linguistic skills involved in comprehending and producing language, and therefore the Hassler et al. study cannot be seen as providing any definitive evidence against the selective facilitation of verbal skills by early musical experience.

Both Costa-Giomi (1999) and Bilhartz et al (2000) randomly assigned groups of children to either a control group or an experimental group that was given music lessons. Costa-Giomi tested the two groups on the Developing Cognitive Abilities Test and found that the experimental group's scores on the spatial subtest were higher than the control group's scores after 1 and 2 years of musical instruction, but not after 3; therefore, the spatial effect was weak and transient at best. No effects of musical experience were found on scores on the verbal subtest, but since the test was a written exam similar to the SAT, stressing verbal comprehension and critical thinking skills, it is unlikely that it was able to assess the on-line speech comprehension and memorization skills most likely to be affected by early musical training and experience.

Bilhartz et al (2000) tested two groups of subjects on several subtests of the Stanford-Binet IQ test, including the Memory for Sentences and Bead Visual Memory subtests. The experimental group showed improved performance on the Visual Memory subtest as compared to the control group, but no such effect was found on the Memory for Sentences subtest. Given the large number of studies that have found a relationship between musical training and short-term auditory memory performance, it is difficult to account for this single anomalous result. It is possible that eight months of musical training was simply not sufficient to cause a significant change in the children's auditory-verbal memory abilities as assessed by these tests.

The results obtained from the comparison group of students in the present study who began practicing gymnastics from an early age suggest that the differences in sequence memory span between musicians and nonmusicians are not the result of gross motor skills or knowledge of and experience in a skilled domain from an early age. While it is true that the musician group consisted of male and female subjects and the gymnastics group was entirely female, it is doubtful that gender was responsible for the differences in performance, as the musicians outperformed the all-male video game players as well. Moreover, given that women slightly out-perform men on verbal working memory tasks (Kramer et al. 1998), one would expect the all-female gymnasts to show higher auditory-verbal spans than the musicians, if effects of gender were driving our results.

Future studies on the effects of early music experience should include multiple comparison groups drawn from other specialized subsets of the population. A 1997 survey of 663 children taking private piano lessons, for example, revealed that the children's parents were mainly college-educated, professional, upper to upper-middle income, Caucasian suburbanites (Duke, Flowers, and Wolfe, 1997). If future studies of the cognitive differences between musicians and non-musicians fail to take into account the many extramusical ways in which musicians may differ from the general population, it will be difficult if not impossible to determine precisely whether or not the observed results are in fact due exclusively to the subjects' selective early experience and activity with music or some other factor.

In summary, we found that skilled musicians displayed significantly longer auditory sequence memory spans than three comparison groups of non-musicians: gymnasts, students enrolled in an introduction to psychology course, and video game players, all of whom were unable to read music. This effect was found to be highly selective and modality-specific in nature and was observed only for the AO condition, and not for the VO and AV conditions of the sequence reproduction task. The present results suggest that music training is linked increased performance on immediate memory span tasks, specifically, memory tasks that require the encoding and reproduction of sequential temporal order information presented via the auditory modality. Our findings suggest that skilled musicians' long history and experience in listening to and playing music may have a selective effect on the processing of auditory temporal sequences in immediate memory. These differences were found to be domain-specific and restricted to the perception, encoding, and maintenance of auditory temporal patterns in immediate memory. 


\section{ACKNOWLEDGEMENTS}

This work was supported by NIH-NIDCD Training Grant T32 DC00012 and NIH-NIDCD Research Grant R01 DC00111 to Indiana University.

\section{REFERENCES}

Atterbury, B. (1985). Musical differences in learning-disabled and normal-achieving readers, aged seven, eight, and nine. Psychology of Music, Vol. 13, 114-123.

Barwick, J., Valentine, E., West, R., \& Wilding, J. (1989). Relations between reading and musical abilities. British Journal of Educational Psychology, Vol. 59, 253-257.

Bilhartz, Terry, Bruhn, Rick, and Olson, Judith (2000). "The effect of early music training on child cognitive development." Journal of Applied Developmental Psychology, Vol. 20, No. 4, 615-636.

Cantor, J., Engle, R.W., \& Hamilton, G. (1991). Short-term memory, working memory, and verbal abilities: How do they relate? Intelligence, Vol. 15, 229-246.

Chan, S., Ho, Y., \& Cheung, M. (1998). Music training improves verbal memory. Nature, Vol. 396, 128.

Costa-Giomi, Eugenia. (1999.) "The effects of Three Years of Piano Instruction on Children's Cognitive Development." Journal of Research in Music Education, Vol. 47, No. 3, 198-212.

Duke, R., Flowers, P., \& Wolfe, D. (1997). Children who study piano with excellent teachers in the United States. Bulletin of the Council for Research in Music Education, Vol. 132, 51-84.

Elbert, T., Pantev, C., Wienbruch, C., Rockstroh, B., \& Taub, E. (1995). Increased cortical representation of the fingers of the left hand in string players. Science, Vol. 270, 305-307.

Gardner, Howard. (1993). Frames of Mind: The Theory of Multiple Intelligences. Basic Books.

Goh, W., \& Pisoni, D. (2003). Effects of lexical competition on immediate memory span for spoken words. The Quarterly Journal of Experimental Psychology: Section A, Vol. 56, 929-954.

Hassler, M., Birbaumer, N., and Feil, A. (1985). "Musical talent and visual-spatial abilities: a longitudinal study." Psychology of Music, Vol. 13, 99-113.

Ho, Y.C., Cheung, M.C. \& Chan, A.S. (2003). Music training improves verbal but not visual memory: Cross sectional and longitudinal explorations in children. Neuropsychology, Vol. 17, 439-450.

House, A., Williams, C., Hecker, M., and Kryter, K. (1965). Articulation-testing methods: consonantal differentiation with a closed-response set. Journal of the Acoustical Society of America, Vol. 37, 158-166.

Huntsinger, C., and Jose, P. (1991.) A test of Gardner's Modularity Theory: a Comparison of Short-term Memory for Digits and Tones. Psychomusicology, Vol. 10, 3-18.

Karpicke, J. \& Pisoni, D.B. (in press). Using immediate memory span to measure implicit learning. Memory and Cognition.

Kilgour, A., Jakobson, L., \& Cuddy, L. (2000). Music training and rate of presentation as mediators of text and song recall. Memory and Cognition, Vol. 28, 700-710. 
Kramer, J., Delis, D., \& Daniel, M. (1988). Sex differences in verbal learning. Journal of Clinical Psychology, Vol. 44, No. 6, 907-915.

Lamb, S., \& Gregory, A. (1993). The relationship between music and reading in beginning readers. Educational Psychology, Vol. 13, 19-28.

Lewellen, M., Goldinger, S., Pisoni, D., \& Greene, B. (1993). Lexical familiarity and processing efficiency: Individual differences in naming, lexical decision, and semantic categorization. Journal of Experimental Psychology: General, Vol. 3, 316-330.

McMahon, O. (1982). A comparison of language development and verbalization in response to auditory stimuli in pre-school age children. Psychology of Music, Vol. 12, 94-105.

Munzer, S., Berti, S., \& Pechmann, T. (2002). Encoding of timbre, speech, and tones: Musicians vs. non-musicians. Psychologische Beitrage, Vol. 44, 187-202.

Nusbaum, H., Pisoni, D., \& Davis, C. (1984). Sizing up the Hoosier Mental Lexicon: Measuring the familiarity of 20,000 words. In Research on Speech Perception Progress Report No. 10 (pp. 357-376). Bloomington, IN: Speech Research Laboratory, Indiana University.

Pisoni, D.B. \& Cleary, M. (2004). Learning, memory and cognitive processes in deaf children following cochlear implantation. In F.G. Zeng, A.N. Popper \& R.R. Fay (Eds.), Springer Handbook of Auditory Research: Auditory Prosthesis, SHAR Volume X. Pp. 377-426.

Schellenberg, E.G. (2004). Music lessons enhance IQ. Psychological Science, Vol. 15, 511-514.

Schlaug, G., Jancke, L., Huang, Y., \& Steinmetz, H. (1995). In vivo evidence of structural brain asymmetry in musicians. Science, Vol. 267, No. 5198, 699-701.

Schlaug, G., Jancke, L., Huang, Y., Staiger, J., \& Steinmetz, H. (1995b). Increased corpus callosum size in musicians. Neuropsychologia, Vol. 33, 1047-1055.

Schneider, P., Scherg, M., Dosch, H., Specht, H., Gutschalk, A., \& Rupp, A. (2002). Morphology of Heschl's gyrus reflects enhanced activation in the auditory cortex of musicians. Nature Neuroscience, Vol. 5, 688-694.

Sommers, M., Kirk, K., \& Pisoni, D. (1997). Some considerations in evaluating spoken word recognition by normal hearing, noise-masked normal hearing, and cochlear implant listeners. I: The effects of response format. Ear and Hearing, Vol. 18, 89-99.

Stallings, L., Kirk, K., Chin, S, \& Gao, S. (2000). Parent word familiarity and the language development of pediatric cochlear implant users. The Volta Review, Vol. 102, 237-258.

Stewart, L., Walsh, V. \& Frith, U. (2004). Reading music modifies spatial mapping in pianists. Perception \& Psychophysics, Vol. 66, 183-195.

Wechsler, D. (1991). Wechsler Intelligence Scale for Children--Third Edition. San Antonio, TX: Psychological Corporation. 